
EDUCATIONAL NEEDS OF RHEUMATOLOGISTS

Z. Ahmad, S. Johnson. Toronto Scleroderma Program, Division of Rheumatology, Department of Medicine, Sinai Health System, University Health Network, University of Toronto, Toronto, Canada

Background: Systemic sclerosis (SSc) is an uncommon, complex and heterogeneous condition, making it challenging to manage. Individual rheumatologists see relatively few cases and patient surveys identify numerous gaps in clinical care. There are no published data on the educational needs of rheumatologists caring for patients with SSc. We aimed to determine rheumatologists' self-rated knowledge and learning needs.

Methods: Survey questions were adapted from the EULAR Recommendations for the Treatment of SSc with reference to patient-identified care gaps. The survey was conducted on paper and on SurveyMonkey (a cloud-based online survey development software program). The target audience was Ontario rheumatologists, serving a population of 13.6 million. We sought to explore self-reported knowledge, experience, attitudes and perceived barriers in caring for SSc patients. Physician demographics and preferred educational methods were also collected. Gaps between perceived and desired knowledge were calculated to identify the greatest unmet learning needs.

Results: One hundred and eighteen responses were received with a response rate of $54 \%$. The greatest unmet learning needs were seen in the management of sexual dysfunction (average gap of 1.4 on a 5 -point scale), pulmonary hypertension (1.1), interstitial lung disease (1.0) and gastrointestinal manifestations of the disease (1.0). The smallest learning gap concerned screening recommendations (0.7). $19 \%$ of rheumatologists agreed with the statement "Scleroderma is an untreatable disease." Agreement with this statement was highest (33\%) among rheumatologists who treat relatively small numbers ${ }^{1-10}$ of scleroderma patients. Conclusions: We have identified several unmet learning needs regarding the management of SSc among rheumatologists. These can be used to inform future educational resources and programs for rheumatologists regarding SSc and to direct further research into their needs.

Disclosure of Interest: None declared

DOI: 10.1136/annrheumdis-2018-eular.1770

\section{AB0826 PROFILE OF MYOSITIS-SPECIFIC ANTIBODIES IN PATIENTS WITH POLYMYOSITIS/DERMATOMYOSITIS AND ASSOCIATION WITH CLINICAL MANIFESTATIONS AND OUTCOME: EXPERIENCE FROM A TERTIARY REFERRAL CENTRE}

Z. Chen, X.-P. Li, X.-M. Li. Rheumatology and Immunology, The First Affiliated Hospital of University of Science and Technology of China, Hefei, China

Background: Myositis-specific antibodies (MSAs) have been found to be associated with distinct clinical phenotype and prognosis in patients with Idiopathic Inflammatory Myopathies.

Objectives: To assess the profile of MSAs in Chinese patients with polymyositis (PM)/dermatomyositis (DM) and explore association of antibody profile with clinical characteristics, laboratory findings and prognosis.

Methods: 90 patients with PM/DM were enrolled. 12 MSAs (mi- $2 \alpha$, mi- $2 \beta$, TIF $1 \gamma$, MDA5, NXP2, SAE1, Jo-1, SRP, PL-7, PL-12, EJ, OJ) were measured by immunoblotting. Associations between antibody profile and clinical manifestations, laboratory data and outcome were determined.

Results: The study population comprised 20 patients with PM and 70 patients with $\mathrm{DM}$, in which $17 \mathrm{DM}$ patients were identified as clinically amyopathic dermatomyositis (CADM). Overall, the most common MSA was anti-ARS (Jo-1/PL-7/PL$12 / \mathrm{OJ} / \mathrm{EJ})(41.1 \%)$, followed by anti-MDA5 (33.3\%), anti-SRP (15.6\%), antiNXP2/anti-SAE1 (13.3\%), anti-TIF1 $\gamma(12.2 \%)$ and anti-mi-2 $\alpha / \mathrm{mi}-2 \beta$ (5.6\%). AntiMDA5 antibody was exclusively seen in DM and CADM patients and the prevalence was higher in CADM than in conventional DM ( $82.4 \%$ vs $30.2 \%, p<0.001)$ Compared with those who were anti-MDA5 negative, patients with positive antiMDA5 had more rapidly progressive interstitial lung disease (ILD) and Gottron sign $(93.3 \%$ vs $61.7 \%, p<0.05 ; 93.3 \%$ vs $38.3 \%, p<0.001)$ as well as lower creatine kinase concentrations (70.0 vs $992.5 \mathrm{IU} / \mathrm{L}, \mathrm{p}<0.001)$. Anti-Jo-1 antibody was more prevalent in $\mathrm{PM}(55.0 \%$ vs $20.8 \%, \mathrm{p}<0.05)$ and patients with positive antiJo-1 demonstrated higher prevalence of mechanic's hands $(18.2 \%$ vs $1.5 \%$, $p<0.05)$. Anti-SRP positive patients had higher creatine kinase levels than those with negative, although there is no statistical significance (1649.5 vs $199.0 \mathrm{IU} / \mathrm{L}$, $\mathrm{p}=0.056)$. Moreover, anti-TIF1 $\gamma$ antibody was associated with more frequent tumour $(18.2 \%$ vs $0.0 \%, \mathrm{p}<0.01)$. In logistic regression analysis, anti-MDA5 $[\mathrm{OR}=9.601,95 \% \mathrm{Cl}(1.940,47.515), \mathrm{p}<0.01]$ was an independent risk factor for ILD. During the follow-up period (median 8 months), 13 patients died, among which 9 were anti-MDA5 positive. The survival time of anti-MDA5 positive patients was significantly less than those who were negative (3.0 vs 11.0 months, $p<0.01$ ). Conclusions: Anti-ARS antibodies are the most common MSAs in Chinese PM DM patients. Anti-MDA5 is predominantly seen in patients with CADM and closely associated with rapidly progressive ILD and high mortality thus serve as a marker of poor prognosis. Anti-TIF1 $\gamma$ positive patients routinely screened for tumours will be of clinical significance.

\section{REFERENCES:}

[1] Clin Rev Allergy Immunol. 2017 Feb;52(1):1-19. doi:10.1007/s12016-0158510-y

[2] Curr Opin Rheumatol. 2016 Nov;28(6):636-44. doi:10.1097/ BOR.0000000000000329

Acknowledgements: We appreciate Dr Rui-Tao Liu for collecting serum samples of PM/DM patients.

Disclosure of Interest: None declared

DOI: 10.1136/annrheumdis-2018-eular.4153

\section{Spondyloarthritis - treatment}

\section{AB0827 HIP ARTHRITIS REMAIN FREE FROM RADIOGRAPHIC PROGRESSION FOR 24 MONTHS FOLLOWING TREATMENT OF ANKYLOSING SPONDYLOARTHRITIS WITH TNF-A INHIBITORS: A PROSPECTIVE STUDY}

A. Djebbari, S. Bouakkaz, S. Bennedjma, S. Oulebsir, M. Houbi, R. Boutefnouchet, A. Beguiret, S. Bounihi, F.Z. Naiji, A. Abiayed. Rheumatology, HCA Hospital, Algiers, Algeria, Algiers, Algeria

Background: Hip involvement is the most frequent extra-spinal arthritic manifes tation of ankylosing spondyloarthritis (AS). It can be severe and may worsen outcomes for patients. There is a large body of high quality evidence for clinica efficacy of TNF- $\alpha$ inhibitors at treating this condition. However, their structural hip benefit remains unknown.

Objectives: In this prospective study undertaken in Algeria, we aimed to evaluate clinical and structural efficacy of TNF- $\alpha$ inhibitor therapies on non-synostosante hip involvement in AS, for a 24 months period.

Methods: This study pursued a follow-up of patients SA using modified New York criteria or ASSAS criteria. Patients were TNF- $\alpha$ inhibitors naîf diagnosed with SA with hip involvement (identified using clinical and/or radiological findings). Patients were treated with one of the following: adalimumab, infliximab or etanercept. Exclusion criteria were: history of tuberculosis, serious infections, hepatitis, neoplasms, other inflammatory conditions, and hip involvement due to any other causes. The following data were collected: clinical rating of hip involvement using the Harris Hip scoring system, biological characteristics (CRP), and radiological characteristics of hip lesions using Bath Ankylosing Spondylitis Radiology Index of the hip (BASRI hip). Specific disease indexes such as BASDAI and BASF were also collected. Follow up was undertaken at the following time periods in months: 0, 3, 6, 12 and 24. Statistical analysis of findings was performed using SPSS 11.0 software

Results: The study recruited a total of 30 patients, 22 males and 8 females. Mean age was $(24,1 \pm 3,1$ years). Bilateral and unilateral hip involvements were identified in $67 \%$ and $33 \%$ of patients, respectively. Mean time for appearance of hip lesions was $(3,9 \pm 2,1$ years). HLAb 27 was present in $30 \%$ of this study population. Baseline characteristics of hips examined have shown an altered function (Harris Hip mean score of $56,1 \pm 5,1$ ) and a relatively advanced structural score (BASRI hip mean score $2,4 \pm 1,1$ ). These scores correlated with high disease activity (BASDAI mean score $5,5 \pm 1,2)$ and a poor mean BASFI score $(5,4 \pm 2,0)$. This was accompanied by a mean CRP score of $22,1 \pm 8,1$. Non-steroidal anti-inflammatory drugs were ineffective. During the 24 months treatment period using TNF- $\alpha$ inhibitors there was a statistically significant improvement in hip scores from the third month onwards with mean Harris Hip scores of 70,3 $\pm 21,5(p<0,001)$, and $81,3 \pm 11,5$ $(p<0,001)$ at months 3 and 6 , respectively. This was maintained until the end of the study period at $94,2 \pm 10,5(p<0,001)$. There have also been statistically significant improvements in BASDAI and BASFI scores as well as CRP (all $p<0,001$ ). Mean BASRI score, however, remained unchanged after 24 months. Treatment was well tolerated, and no significant complications (e.g. serious infections or tuberculosis) were observed. No prosthesis was indicated for any patient

Conclusions: Hip involvement is associated with severe and rapidly evolving AS. In this study, we have demonstrated improved outcomes and stability of radiographic lesions of hip arthritis for a period of 24 months, when SA was treated with TNF- $\alpha$ inhibitors.

Disclosure of Interest: None declared 